\title{
Cutaneous Langerhans Cell Sarcoma: A Case Report
}

\section{Maurizio Giuliani*, Alessandro Marinucci, Loredana Melchiorri and Giovanni Zoccali}

Department of Life, Health \& Environmental Sciences, University of L'Aquila, Plastic and Reconstructive Section, P.le Salvatore Tommasi,1 - 67100 - L'Aquila, Italy

Background: Langerhans Cells are a subset of antigen-presenting dendritic cells belonging to the histiocytic system and are classified into Langerhans cell histiocytosis and Langerhans cell sarcoma. Histiocyte tumors are among the more uncommon neoplasms affecting lymphoid tissues, probably representing less than $1 \%$ of all malignancies involving lymph nodes or soft tissues. Langerhans cell sarcoma can be limited to the skin, or it can spread to other organs. To the best of our knowledge, primary cutaneous Langerhans cell sarcoma without any extracutaneous associations is extremely rare; we describe a young male patient with this rare condition.

Materials and Method: After the surgical excision the specimen underwent to Immunohistochemistry staining showing that the tumor cells were diffusely positive for vimentin, S-100, CD68, CD1a, and langerin but negative for melan-A and pan-cytokeratin. The Ki-67 proliferating index was high at approximately $12 \%$.

Discussion and Conclusion: Histiocytoses are rare neoplasms with a highly unpredictable clinical course ranging from spontaneous regression to highly aggressive, even fatal, disease. Cutaneous Langerhans cell sarcoma, with its rareness in skin and its poorly differentiated morphologic features, shares a differential diagnosis with other epithelial or mesenchymal skin neoplasms nonetheless the typical immunophenotype features langerin, CD1a, and S-100 are helpful to achieve an accurate diagnosis of these dendritic-cell tumors.

\section{Introduction}

Langerhans Cells (LC) is a subset of antigen-presenting dendritic cells belonging to the histiocytic system. The World Health Organization (WHO) classifies LC tumors into Langerhans cell histiocytosis (LCH) and Langerhans cell sarcoma (LCS). LCS is a rare neoplastic proliferation of LC with overtly malignant cytologic features. The condition can present de novo, or it can derive from a previous $\mathrm{LCH}$ $[1,2]$. Histiocyte tumors are among the more uncommon neoplasms affecting lymphoid tissues, probably representing less than $1 \%$ of all malignancies involving lymph nodes or soft tissues [3-4]. They can occur at any age, although incidence peaks around the fourth decade of life. Skin and the underlying soft tissues are the most common sites of tumor origin, but the involvement of bone, lung, gallbladder, and peritoneal lymph nodes has also been described $[5-7,1]$.

The diagnosis of cutaneous LCS can be difficult: metastatic cancer, malignant melanoma, anaplastic large-cell lymphoma, and myeloid sarcoma show similar microscopic features. LCS can be limited to the skin, or it can spread to other organs [8]. To the best of our knowledge, primary cutaneous LCS without any extracutaneous associations is extremely rare; we describe a young male patient with this rare condition.

\section{Case Report}

A 25 year-old Caucasian male, otherwise healthy, was referred to a dermatologist for a single rapidly growing lesion on his left leg. The $12-\mathrm{mm}$ nodule was firm and erythematous, with central ulceration (Figure1). He reported no weight loss and there was no palpable lymphadenopathy or organomegaly. Surgical resection of the skin lesion was performed without preoperative skin biopsy.

The surgical specimen was noted to be a subcutaneous nodule, yellowish in color. The nodule was fixed in $10 \%$ neutral buffered formalin and then embedded in paraffin and sectioned in $4-\mu \mathrm{m}$ thick slices. The slices were then stained with hematoxylin and eosin Immunohistochemical analyses were performed using the ChemMate
Envision/HRP Kit (Dako, Glostrup, Denmark). Antibodies against vimentin, S-100 protein, cluster of differentiation CD 68, CDla, langerin, pan-cytokeratin, melan-A, and Ki-67 were obtained from DakoCytomation (Dako, Glostrup, Denmark).

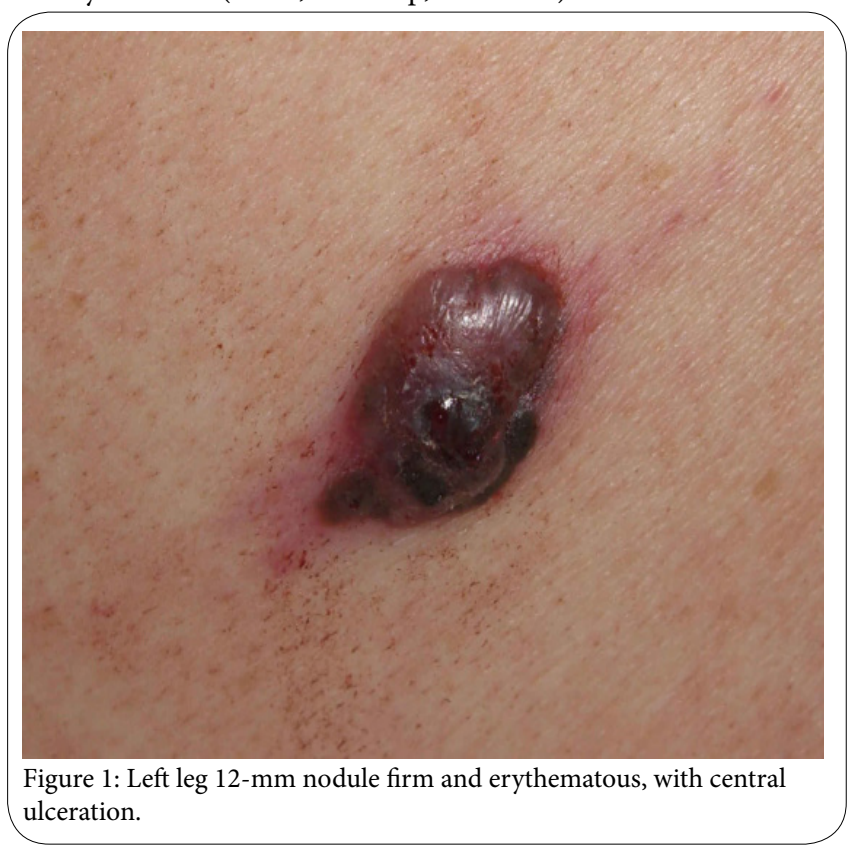

${ }^{*}$ Corresponding Author: Dr. Maurizio Giuliani, Department of Life, Health \& Environmental Sciences, University of L'Aquila, Plastic and Reconstructive Section P.le Salvatore Tommasi, 1 - 67100 - L'Aquila, Italy; E-mail: maurizio.giuliani@cc.univaq.it

Citation: Giuliani M, Marinucci A, Melchiorri L, Zoccali G (2016) Autoimmune Pulmonary Alveolar Proteinosis: Still Cloudy with a Chance of Understanding. Int J Surg Surgical Porced 1: 101. doi: http://dx.doi.org/10.15344/ijssp/2016/101

Copyright: (C) 2016 Giuliani et al. This is an open-access article distributed under the terms of the Creative Commons Attribution License, which permits unrestricted use, distribution, and reproduction in any medium, provided the original author and source are credited. 
Citation: Giuliani M, Marinucci A, Melchiorri L, Zoccali G (2016) Autoimmune Pulmonary Alveolar Proteinosis: Still Cloudy with a Chance of Understanding. Int J Surg Surgical Porced 1: 101. doi: http://dx.doi.org/10.15344/ijssp/2016/101

Page 2 of 3

\section{Pathological Findings}

The irregularly shaped nodule showed a central ulceration, with diffuse infiltration of large tumor cells into the dermis. The tumor cells had significant malignant cytological features: they exhibited an irregular shape with abundant eosinophilic, sometimes clear, cytoplasm and large, irregular shaped nuclei with a lobulated or dented appearance. Some cells displayed a longitudinal nuclear groove and prominent nucleoli. A high mitotic rate (more than 30 mitoses per 10 high-power fields) was observed. Moderate inflammatory infiltration consisting of neutrophils, plasma cells, eosinophils, lymphocytes, and macrophages was observed, interspersed with the tumor cells (Figure 2).

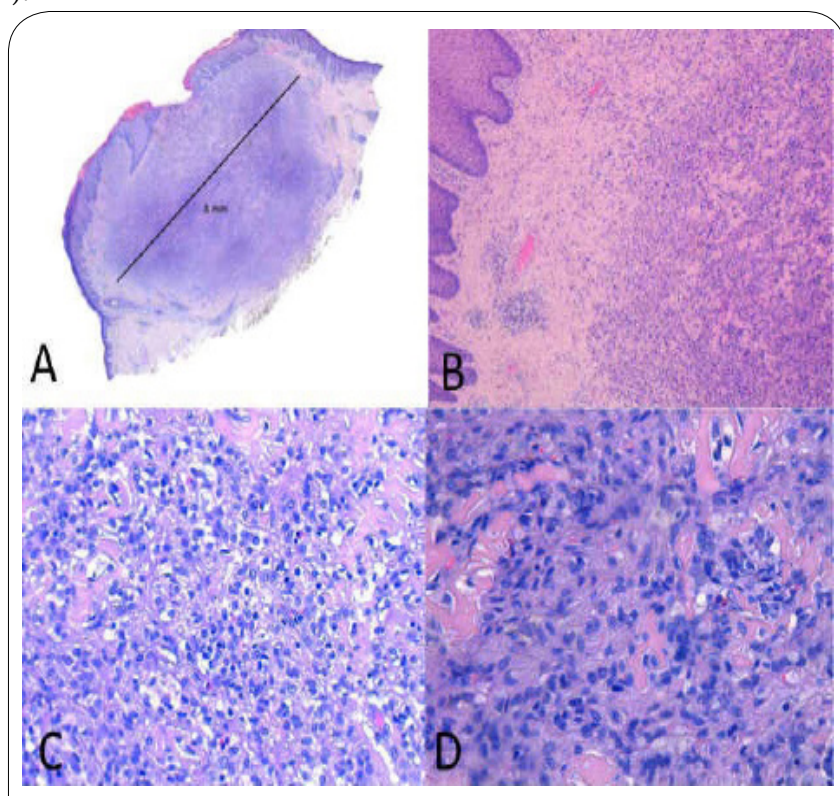

Figure 2: A (sub-macroscopic view) - B (1X): irregularly shaped nodule with central ulceration and diffuse infiltration of large tumor cells into the dermis. C (20X) - D (40X): tumor cells exhibited an irregular shape with abundant eosinophilic, sometimes clear, cytoplasm and large, irregular shaped nuclei with a lobulated or dented appearance.

\section{Immunohistochemical Staining and Results}

Immunohistochemistry staining showed that the tumor cells were diffusely positive for vimentin, S-100, CD68, CD1a, and langerin but negative for melan-A and pan-cytokeratin. The Ki-67 proliferating index was high at approximately $12 \%$.

The nodular growth pattern, significantly malignant cytological features, high mitotic index, and immunohistochemical aspects led us to the diagnosis of a cutaneous LCS, according to the WHO diagnostic criteria (Figure 3, 4).

\section{Discussion}

Histiocytoses are rare neoplasms with a highly unpredictable clinical course ranging from spontaneous regression to highly aggressive, even fatal, disease. LCS has the potential to progress to leukemia $[3,8]$. The typical sites for LCS development are skin, bone, and lymphoid tissues. The diagnostic criteria of LCS include the presence of cells that have both characteristics of LC and typical histological features of tumor cells. The typical immunophenotype features langerin, CD1a, and S-100 are helpful to achieve an accurate diagnosis of these dendriticcell tumors. Nonetheless, the diagnosis of LCS can be difficult:

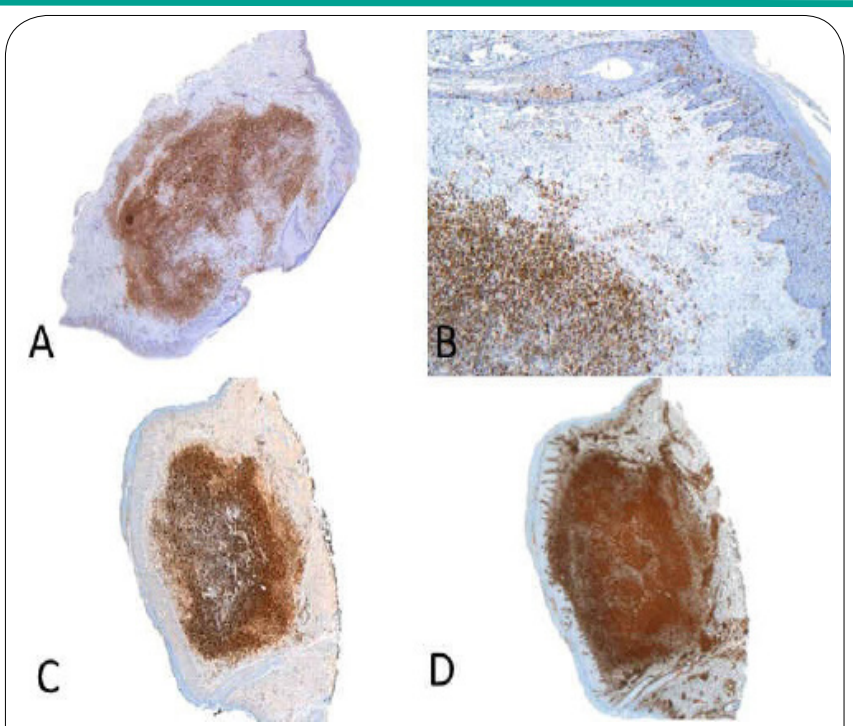

Figure 3: A (sub-macroscopic view) - B (4X): Langerhans tumoral cells CD1a +. C (sub-macroscopic view) : Langerhans tumoral cells S100 +. D (sub-macroscopic view): Langerhans tumoral cells Vimentin + .

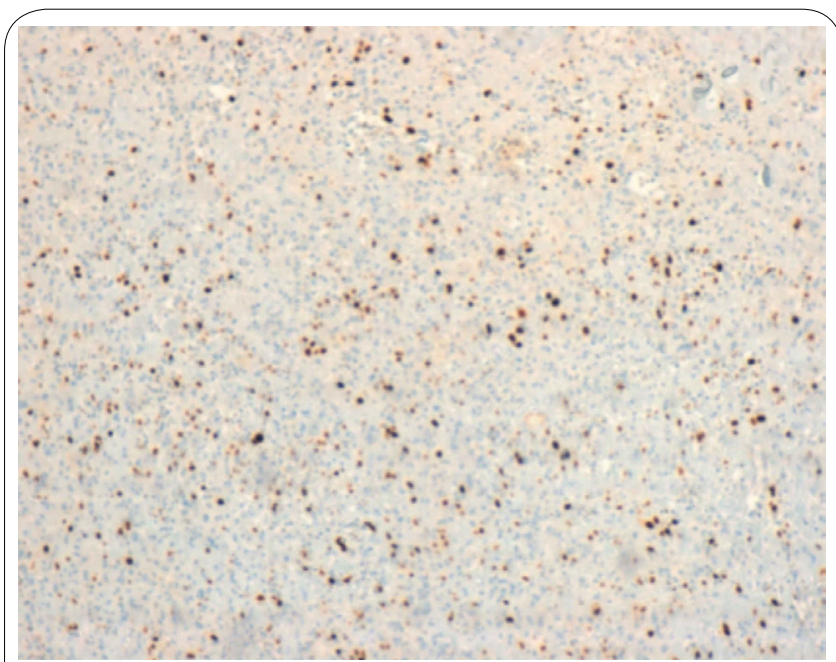

Figure 4: Ki-67 proliferating index approximately $12 \%$.

tumor cells with poorly differentiated or atypical morphology might occasionally lose the distinctive immunophenotype.

According to the WHO classification system, tumors of the dendritic-cell lineage include follicular dendritic-cell tumors, interdigitating dendritic-cell tumors, LC tumors, and other rare dendritic-cell tumors. LC and interdigitating dendritic cells share a common hematopoietic CD34+ precursor; in contrast, follicular dendritic cells do not have a hematopoietic origin. LC tumors show expression of both CD1a and S-100 protein, while interdigitating cells are positive for S-100 but negative for CD1a. Follicular dendritic cells express CD21 consistently, but never express CD1a [9].

Cutaneous LCS, with its rareness in skin and its poorly differentiated morphologic features, shares a differential diagnosis with other epithelial or mesenchymal skin neoplasms. All of these diseases exhibit skin lesions and a frankly malignant cytologic appearance with a highly aggressive clinical course and a poor prognosis. However, cutaneous squamous-cell carcinoma and metastatic cancer 
both display an obvious nest structure with an epithelial phenotype including pan-cytokeratin, cytokeratin CK 7, or CK20. Melanoma might share S-100 protein positivity with LCS, but it also expresses other melanocytic markers such as human melanoma black (HMB) 45 and melan-A. Anaplastic large-cell lymphomas are positive for CD30 and epithelial membrane antigen (EMA) and may show positivity for anaplastic lymphoma kinase (ALK). Myeloid leukemia can originate in the skin, and CD68 and lysozyme positivity may be observed, which could make this condition difficult to distinguish from LCS. The presence of myeloid-specific markers, such as myeloperoxidase (MPO), CD117, CD99, and CD34, should be helpful in differentiating cutaneous myeloid leukemia from cutaneous myeloid sarcoma [10]. In our patient, the tumor cells strongly expressed CD1a, S-100 protein, and langerin, markers specific for LC. These findings argued against other cutaneous hematological neoplasms.

\section{Conclusion}

In conclusion, LCS is extremely rare and only a few cases of LCSs with exclusive cutaneous involvement have been reported in the literature. Our patient represents this rare category of disease. The diagnosis of primary cutaneous LCS is difficult and should be made cautiously, particularly in patients with clinically localized disease who lack extracutaneous manifestations.

\section{Competing Interests}

The authors declare that they have no competing interests.

\section{Author Contributions}

Maurizio Giuliani: wrote the paper, had drawn the study and gave the final approval.

Alessandro Marinucci: performed the laboratory analysis, had cooperated in study drawing.

Loredana Melchiorri: performed the laboratory analysis, had cooperated in study drawing.

Giovanni Zoccali: performed the literature review and revisited the manuscript.

\section{References}

1. Zhao G, Luo M, Wu ZY, Liu Q, Zhang B, et al. (2009) Langerhans cell sarcoma involving gallbladder and peritoneal lymph nodes: a case report. Int J Surg Pathol 17: 347-353.

2. Lee JS, Ko GH, Kim HC, Jang IS, Jeon KN, et al. (2006) Langerhans cel sarcoma arising from Langerhans cell histiocytosis: a case report. J Korean Med Sci 21: 577-580.

3. Shao H, Xi L, Raffeld M, Feldman AL, Ketterling RP, et al. (2011) Clonally related histiocytic/dendritic cell sarcoma and chronic lymphocytic leukemia/ small lymphocytic lymphoma: A study of seven cases. Mod. Pathol 24: 1421-1432.

4. Who Classification of Tumours of Haematopoietic and Lymphoid Tissues (2008) International Agency for Research on Cancer. Lyon, France.

5. Kawase T, Hamazaki M, Ogura M, Kawase Y, Murayama T, et al. (2005) CD56/NCAM-positive Langerhans cell sarcoma:a clinicopathologic study of 4 cases. Int J Hematol 81: 323-329.

6. Jülg BD, Weidner S, Mayr D (2006) pulmonary manifestation of a Langerhans cell sarcoma: case report and review of the literature. Virchows Arch 448: 369-374.

7. Ben-Ezra J, Bailey A, Azumi N, Delsol G, Stroup R, et al. (1991) Malignant histiocytosis X. A distinct clinicopathologic entity. Cancer 68: 1050-1060.

8. Itoh H, Miyaguni H, Kataoka H, Akiyama Y, Tateyama S, et al. (2001) Primary cutaneous Langerhans cell histiocytosis showing malignant phenotype in an elderly woman: report of a fatal case. J Cutan Pathol 28: 371-378.

Int J Surg Surgical Porced
9. Weiss LM, Chan JKC, Fletcher CDM (2008) Other rare dendritic cell tumors. In: Swerdlow SH, Campo E, Harris NL, Jaffe ES, Pileri SA et all, (Ed. IARC, W.) WHO classification of tumors pathology and genetics of tumors of haematopoietic and lymphoid tissues $\mathrm{HO}$ press.

10. Yang Li, Bin Li, Xiao-ying Tian, Zhi Li. (2013) Unusual cutaneous Langerhans cell sarcoma without extracutaneous involvement. Diagn Path 8: 20. 